\title{
Research on E-shape Magnetic Core Based Self-differential Mode pick-up Coil and Its Application for Fine Crack Non-destructive Testing
}

\author{
Xiaojie Xu, Zhanbin Zhang, Yueling Jia \\ Institute of Information and Navigation, \\ Air Force Engineering University, \\ Xi’an, China
}

\begin{abstract}
Remote field eddy current was proved to be an effective non-destructive testing method for tubular structure such as tubes and pipes. Further applications of remote field eddy current testing were restricted by disadvantages such as weak signal, over-long sensor, and poor ability to detect fine crack especially axial crack. Differential mode pick-up coil could make up the disadvantages to some extent. On the basis of analyzing advantages and disadvantages of conventional differential mode pick-up coil, a self-differential mode pick-up coil based on E-shape magnetic was proposed and verified by simulation and experiment. Conclusions could be deduced by simulation and experiment results: the performance of remote field eddy current could be improved significantly by using self-differential mode pick-up coil, and adverse effect which conventional differential mode usually brought could be avoided.
\end{abstract}

Keywords-remote field eddy current; self-differential mode; pick-up coil; finite element simulation; detection sensitivity

\section{INTRODUCTION}

Remote field eddy current (RFEC) technique recently draws more and more attention in non-destructive testing of tubes and pipes for its advantages as follows [1]. Almost equal sensitivity to defects in inner diameter (ID) and outer diameter (OD), easy defect characterization for the linear relation between sensor signal and wall depth (WD), and insensitivity to lift-off or wobble.

However, further applications of remote field eddy current testing were restricted by disadvantages such as weak signal, over-long sensor. In this paper, we investigate a novel self-differential mode pick-up coil based on E-shape magnetic core to overcome these disadvantages, and after analyzing and declaring the basic principle of it by finite element method, experiment installation and a real stress corrosion crack is used to verify it.

\section{REMOTE FIELD EDDY CURRENT SIMULATION AND ANALYSIS}

\section{A. Principle of Remote Field Eddy Current}

Figure 1 shows the conventional schematic diagram of remote field eddy current, which is dependent on two different coupled path when electromagnetic wave propagate in the pipe [2].

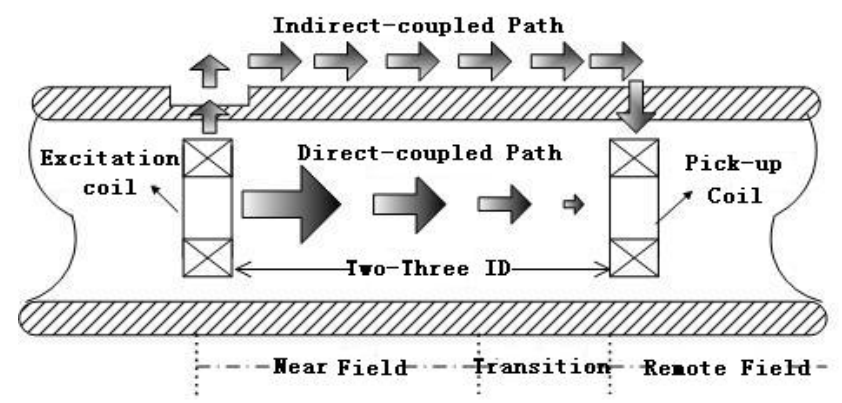

Figure 1. Schematic diagram of remote field eddy current.

\section{B. Crack Detection Ability of Conventional Pick-up Coil}

Figure 2 shows conventional remote field eddy current sensor usually adopts a coaxial solenoid coil excited by sinusoid to generate exciting field, and adopts another solenoid coil placed at about 2-3 times pipes inner diameter to pick up field [3].

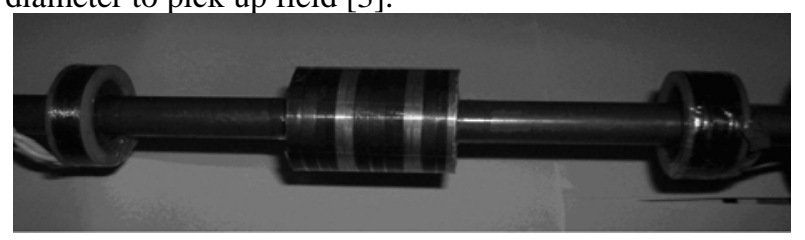

Figure 2. Conventional sensor prototype of RFEC testing.

To verify detection ability and sensitivity of conventional sensor, two classes defects are manufactured in a section of ferromagnetic tube with $70 \mathrm{~mm}$ inner diameter, $82 \mathrm{~mm}$ outer diameter, and $6 \mathrm{~mm}$ wall thickness. First class defects shown in Figure 3 include: circumferential and axial crack with $10 \mathrm{~mm}$ length and 2 $\mathrm{mm}$ width, circular defect with $6 \mathrm{~mm}$ diameter; second class defect shown in Figure 4 include: circumferential and axial crack with $10 \mathrm{~mm}$ length and $0.5 \mathrm{~mm}$ width, circular defect with $3 \mathrm{~mm}$ diameter. All defects are manufactured with depth of $3 \mathrm{~mm}$, equal to $50 \%$ tube wall thickness. 


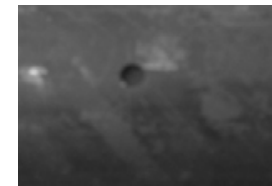

(a)

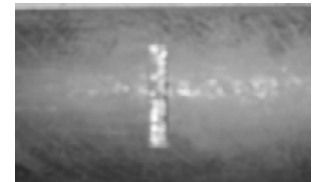

(b)

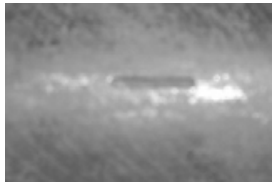

(c)

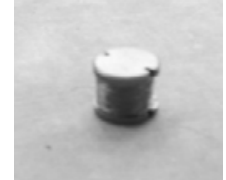

(d)

Figure 3. Photography of first class defects and pick-up coil. (a) Circular, (b) circumferential, (c) axial, (d) pick-up coil.

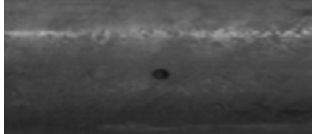

(a)

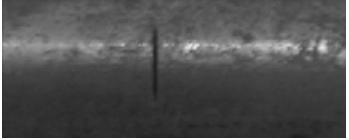

(b)

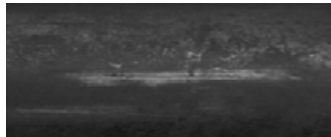

(c)

Figure 4. Photography of second class defects. (a) Circular, (b) circumferential, (c) axial.

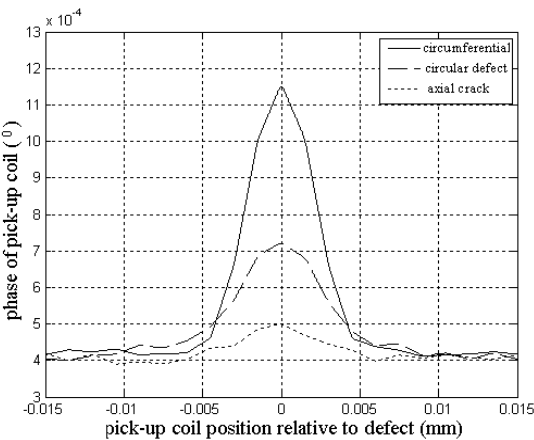

(a)

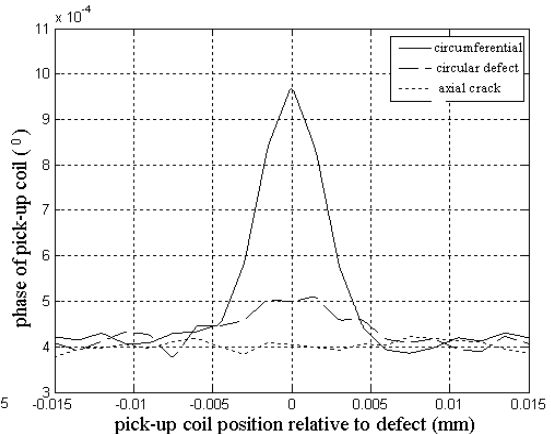

(b)

Figure 5. Experiment results for defects. (a) First class, (b) second class.

Figure 5(a) shows the experiment results of first class defects. All defects can be detected and identified clearly, although sensitivity to axial crack is relative lower than circumferential crack and circular defect. However, with reduction of size of defect, all of defects detection ability decreases, especially for axial crack. Figure 5(b) shows experiment results of second class defects. Amplitude of axial cracks reduce too low to detect it.

\section{DIFFERENTIAL MODE PICK-UP COILS}

In view of the above mentioned, the detection ability and sensitivity to fine crack are very poor, especially for axial cracks. It is mainly because of that remote field eddy current is a relative weak signal system, using an absolute mode pick-up coil is not easily to detect the disturbance of fine crack. Differential mode pick-up coil can be used to improve the detection sensitivity; due to back-up level can be removed from the detection signal $[4,5]$.

Figure 6 shows three types of differential mode pickup coils. Adopting two rod-shape pick-up coils shown in Figure 6(a) is the most popular differential mode used for eddy current nondestructive testing, but it is susceptible to jitter and off-center of sensor and it is difficult to ensure uniformity of the two windings. Adopting two U-shape pick-up coils shown in Figure 6(b) can reduce the influence of jitter and off-center of sensor due to windings sharing same magnetic path, but it is still difficult to deal with uniformity of two windings.

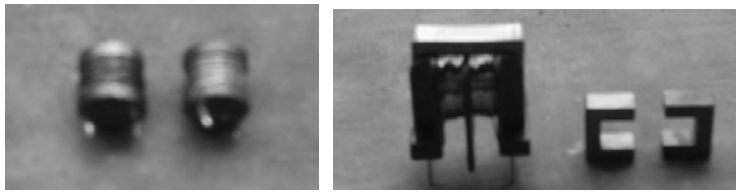

Figure 6. (a) Conventional differential, (b) U-shape differential.

\section{A. Principle of Self-differential Mode Based on E-shape} Magnetic Core

Adopting pick-up coil with E-shape magnetic core shown in Figure 7 is a new type differential mode, which is proposed firstly to detect cracks in aircraft multilayer structure [6], and is named as "self-differential."

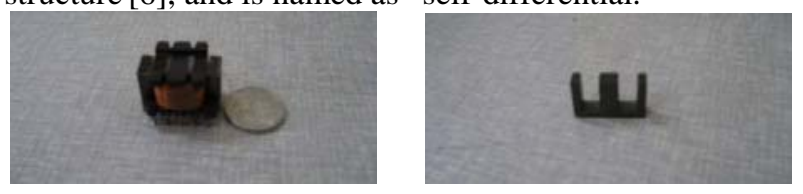

Figure 7. Photography of E-shape pick-up coil.

Figure 8 shows basic principle of it. The pick-up coil winds on central axis of the E-shape magnetic core. In symmetric case which means defect free, there will be no magnetic flux enters the central axis, because of that magnetic flux always follows the path which has minimum reluctance according to magnetic path theory. When one of the side shafts of pick-up coil pass through a crack, because of the stronger magnetic field intensity around crack, the symmetry will be broken, then a part of 
magnetic flux will enter central axis from bottom to up and is picked up by the winding. When another side shaft pass through the crack, a part of magnetic flux will enter central axis from opposite direction.

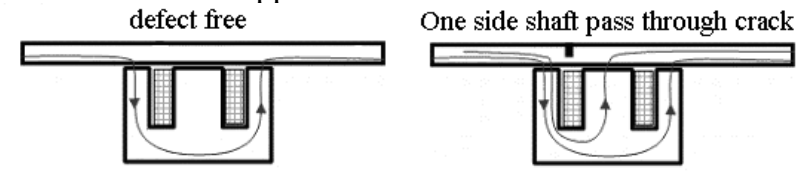

another side shaft pass through crack

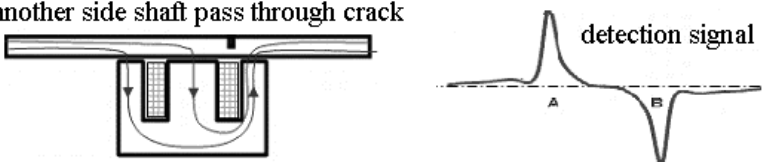

Figure 8. Principle of E-shape pick-up coil.

B. Simulation of Self-differential Mode Based on E-shape Magnetic Core

When excitation frequency is low, the displacement current can be neglected, and the basis governing Maxwell equation will be reduced to following equations [3]:

$\nabla \times \vec{H}=\vec{J}+\vec{J}_{S}$

$\nabla \times \vec{E}=-\frac{\partial \vec{B}}{\partial t}$

$\nabla \cdot \vec{B}=0$

where $\vec{J}_{S}$ is source current density, $\vec{J}$ is eddy current density, $\vec{B}$ and $\overrightarrow{\boldsymbol{H}}$ are magnetic flux density and magnetic field intensity, $\overrightarrow{\boldsymbol{E}}$ is electric field intensity.

Introducing magnetic potential vector $\vec{A}$, which is governed by $\overrightarrow{\boldsymbol{B}}=\nabla \times \overrightarrow{\boldsymbol{A}}$, the final governing equation is derived from Eqs. (1)-(3):

$\nabla \times \frac{1}{\mu} \nabla \times \vec{A}=-\sigma \frac{\partial \vec{A}}{\partial t}+\vec{J}_{S}$

In harmonic excitation, whose angular frequency is $\omega$, and considering coulomb gauge $\nabla \cdot \vec{A}=0$, Eq. (5) is rewritten as:

$$
\left(\frac{1}{\mu}\right) \nabla^{2} \vec{A}=-\vec{J}_{S}+j \omega \sigma \vec{A}
$$

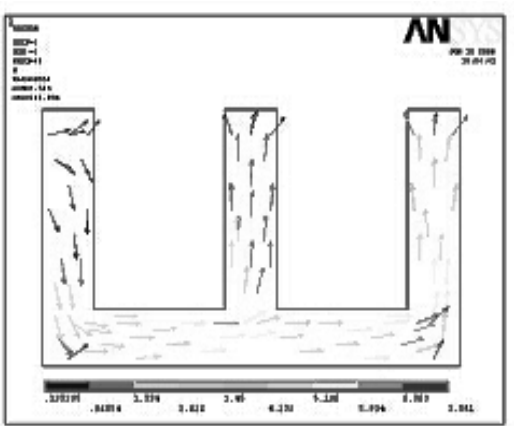

Then we obtain magnetic potential vector $\vec{A}$ by using FEM to mesh and work out Eq. (5), therefore magnetic flux density $\vec{B}$ and other wanted quantity can be deduced from it.

TABLE I. PARAMETERS OF TUBE AND RFEC SENSOR

\begin{tabular}{|c|c|}
\hline \multicolumn{2}{|c|}{ Tube } \\
\hline Outer diameter [mm] & 42 \\
\hline inner diameter [mm] & 36 \\
\hline Wall thickness [mm] & 3 \\
\hline Relative permeability & 70 \\
\hline Conductivity [s/m] & $0.5 \times 10^{7}$ \\
\hline OD defect depth [mm] & 0.6 \\
\hline OD defect width [mm] & 2 \\
\hline Sensor \\
\hline Exciter current density coil [A/m $\left.{ }^{2}\right]$ & $0.3 \times 10^{7}$ \\
\hline Frequency of excitation coil $(\mathrm{Hz})$ & 30 \\
\hline
\end{tabular}
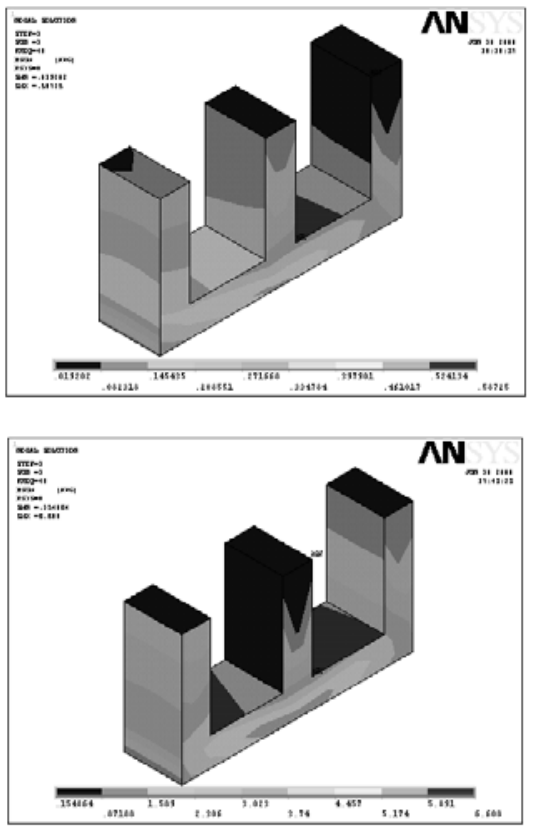

Figure 9. Contour plot of magnetic flux density.

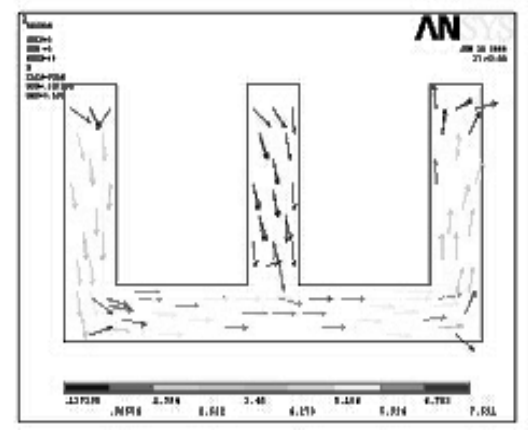

Figure 10. Vector plot of magnetic flux density. 
Table I lists the simulation parameters.

Figures 9 and 10 show the contour plot of and the vector plot of magnetic flux density. From the contour and vector plot, the principle is declared clearly: when defect free, there is no magnetic flux enter central axis. But when one of the shaft pass through crack, magnetic will enter the central axis, and picked up by windings, which means "self-differential mode" pick-up coil.
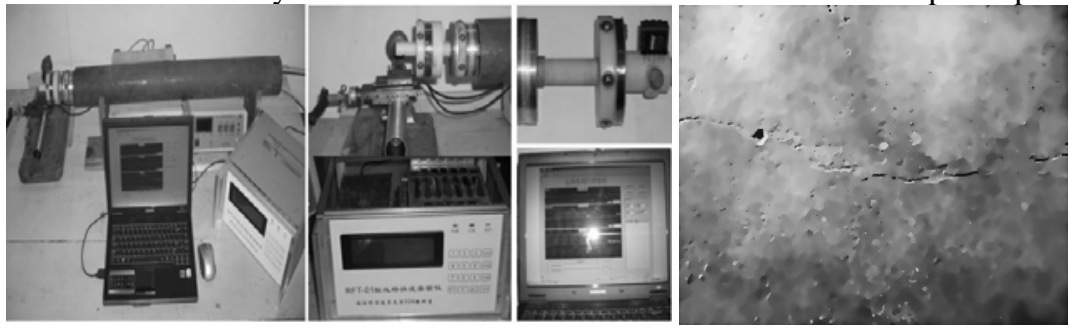

Figure 11. Photography of experiment installation.

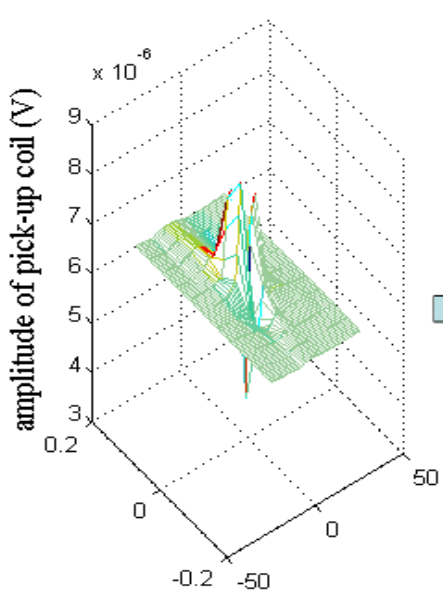

(a) 3D plot

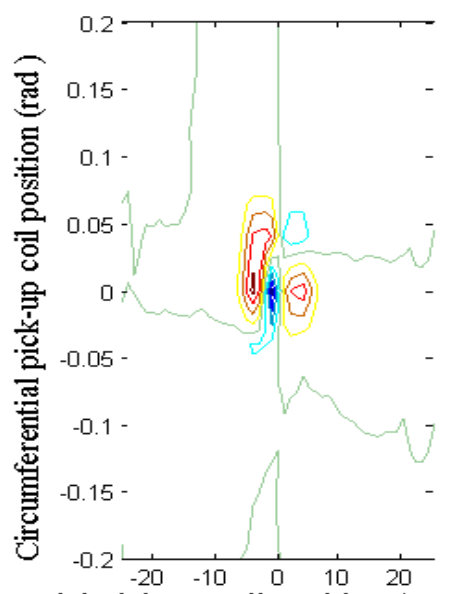

Axial pick-up coil position (mm)

(b) contour plot

Figure 12. Amplitude of pick-up coil.

\section{EXPERIMENT RESULTS}

Figure 11 shows the experiment installation, sensor, and crack. The sensor consists of exciting coil, centering device using to reduce influence of sensor jitter or offcenter, self-differential mode pick-up coil, and shielding facility using to reduce length of sensor. The test specimen is a real tube with a stress corrosion crack on its out surface, which cannot be detected using the conventional absolute pick-up coil.
Figures 12 and 13 experiment results of the stress corrosion crack. From the 3D plot of amplitude of pick-up coil shown in Figure 11(a), the stress corrosion crack can be detected clearly, but it is not easily to determine the shape of the crack even in the contour plot. Fortunately, we can use the contour plot of phase shown in Figure 12(b) to identify shape of the crack, for phase is usually chosen to determine defect in remote field eddy current. 


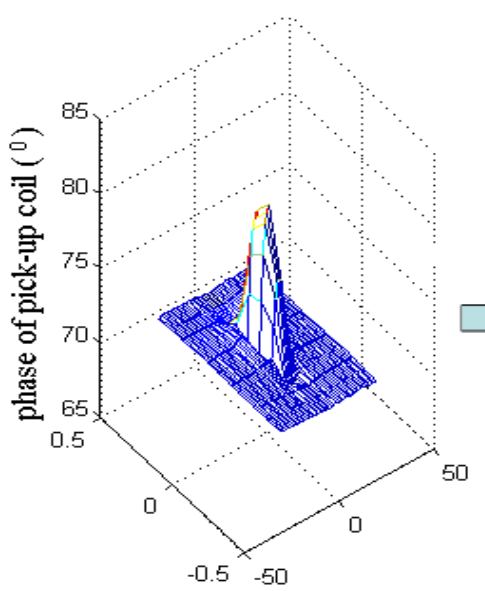

(a) $3 \mathrm{D}$ plot

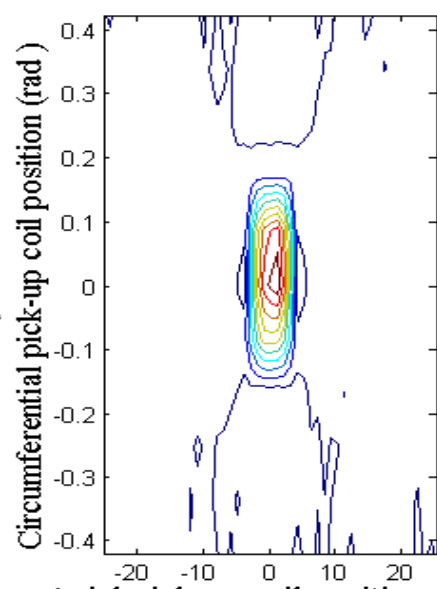

Axial pick-up coil position (mm)

(b) contour plot

Figure 13. Phase of pick-up coil.

\section{CONCLUSIONS}

The pick-up determines the testing ability and sensitivity of remote field eddy current. Adopting conventional differential mode pick-up coil can eliminate some disadvantages of RFEC, but inconsistency of the two windings will cause another problem such as large error and more susceptible to off-center or wobble. Selfdifferential mode pick-up coil can avoid the adverse effect for the reason of only one winding needed to realize differential mode.

\section{ACKNOWLEDGMENTS}

The research work is supported by National Natural Science Foundation of China under Grant No. 51107148 and The Natural Science Foundation of Shanxi Province (2011JQ7006).

\section{REFERENCES}

[1] Javier García Martín, Jaime Gómez Gil, Ernesto Vázquez-Sánchez, non-destructive techniques based on eddy current testing. Sensors, 11(8), pp. 2525-2565, 2011.

[2] Noriyasu Kobayashi, Souichi Ueno, Satoshi Nagai, et al., Remote field eddy current testing for steam generator inspection of fast reactor. Nuclear Engineering Research, 241(11), pp. 4643-4648, 2011.

[3] Chen Zhen Mao, Mihai Rebican, Kenzo Miya, Three dimensional simulation of remote field ECT using the Ar method and a new formula for signal calculation. Research in Non-Destructive Evaluation, 16(1), pp. 35-53, 2005.

[4] Luis S. Rosado, Telmo G. Santos, A differential planar eddy currents probe: Fundamentals, modeling and experimental evaluation. NDT \& E International, 51(1), pp. 85-93, 2012.

[5] R. Robaina, T.A. Héctor, Planar coil-based differential electromagnetic sensor with null-offset. Sensors \& Actuators A, 164(1), pp. 15-21, 2010.

[6] Yushi Sun, Dennis Roach, Harry Zhu, New advances in detecting cracks in raised-head fastener holes using rotational remote field eddy current technique. In ASNT Fall Conference, Ohio, pp. 17-21, 2005. 